\title{
On Eberlein compactifications of metrizable spaces
}

\author{
by \\ Takashi Kimura (Urawa) and Kazuhiko Morishita (Ashikaga)

\section{Dedicated to Professor Takao Hoshina on his sixtieth birthday}

\begin{abstract}
We prove that, for every finite-dimensional metrizable space, there exists a compactification that is Eberlein compact and preserves both the covering dimension and weight.
\end{abstract}

1. Introduction. In this paper, we assume that all spaces under consideration are Tikhonov. Usual and undefined terms can be found in [1], [3] and [4]. It is well known that for every separable metrizable space, there exists a dimension-preserving metrizable compactification, and for every Tikhonov space, there exists a compactification preserving both the dimension dim and weight. However, obviously, no non-separable metrizable space has a "metrizable" compactification. It seems a natural desire to find some "nice" class of compact spaces in the sense that not only does every space in the class have "nice" topological properties, but also for every metrizable space, there exists a compactification in the class preserving both the dimension dim and weight.

A compact space $E$ is said to be Eberlein compact if $E$ is homeomorphic to a subset of a Banach space with its weak topology. The class of Eberlein compact spaces plays an important role in functional analysis; and moreover, it is stable in the sense that it is closed under taking countable products, closed subsets and continuous images of spaces in this class (see [1]). It is known that some non-metrizable spaces belong to this class; in fact, the onepoint compactifications of discrete spaces are Eberlein compact. However, the difference between the class of compact metrizable spaces and the class of Eberlein compact spaces seems small in the sense that every Eberlein compact space $E$ has the following properties (see [1]):

2000 Mathematics Subject Classification: 54C35, 54D35, 54F45.

Key words and phrases: Eberlein compact, dimension, compactification, hedgehog, residual. 
- $E$ contains a metrizable dense $G_{\delta^{-} \text {-subset, }}$

- $E$ is a Fréchet space,

- $n w(A)=d(A)$ for every subset $A$ of $E$, and

- $c(E)=w(E)$.

For a space $X$, an Eberlein compact space $E$ is said to be an Eberlein compactification of $X$ if $E$ contains $X$ as a dense subset. Arkhangel'skiu proved that every metrizable space has an Eberlein compactification (see [1]). Moreover, Dimov presented a characterization theorem for spaces that have Eberlein compactifications (see [2]). Our purpose is to prove the following two theorems.

Theorem 1.1. Let $M$ be a finite-dimensional metrizable space. There exists an Eberlein compactification $E$ of $M$ such that $\operatorname{dim} E=\operatorname{dim} M$ and $w(E)=w(M)$.

TheOREM 1.2. Let $M$ be an S-weakly infinite-dimensional metrizable space. There exists an $S$-weakly infinite-dimensional Eberlein compactification $E$ of $M$ with $w(E)=w(M)$.

For the definition of S-weakly infinite-dimensionality, see [4, Problem 6.1.E(a), Remark, p. 310]. We abbreviate "S-weakly infinite-dimensional" to S-w.i.d.

2. Proofs of the theorems. First, we set our terminology and notation. The symbols $\mathbb{I}, \mathbb{Q}$ and $\mathbb{N}$ denote the closed unit interval $[0,1]$, the set of rational numbers and the set of positive integers respectively. Let $\tau$ be an infinite cardinal. For every $\alpha \leq \tau$, we put $\mathbb{I}_{\alpha}=\mathbb{I} \times\{\alpha\}$. Moreover, we set

$$
T(\tau)=\bigcup_{\alpha<\tau} \mathbb{I}_{\alpha} \text { and } T^{*}(\tau)=\bigcup_{\alpha \leq \tau} \mathbb{I}_{\alpha}
$$

For $(x, \alpha),(y, \beta)$ in $T^{*}(\tau)$, we define an equivalence relation $\sim$ as follows:

$$
(x, \alpha) \sim(y, \beta) \Leftrightarrow\left\{\begin{array}{l}
x=y=0, \text { or } \\
x=y \text { and } \alpha=\beta .
\end{array}\right.
$$

Denote by $S^{*}(\tau)$ the set of all equivalence classes of $\sim$, by q the natural mapping of $T^{*}(\tau)$ to $S^{*}(\tau)$, by p be the mapping from $S^{*}(\tau)$ to $\mathbb{I}$ defined by $\mathrm{p}(\mathrm{q}(x, \alpha))=x$, and by $\lambda$ the mapping from $S^{*}(\tau) \backslash\{\mathrm{q}(\{(0, \alpha) \mid \alpha \leq \tau\})\}$ to $\tau+1$ defined by $\lambda(\mathrm{q}(x, \alpha))=\alpha$. For every $\mathrm{q}(x, \tau)$ in $\mathrm{q}\left(\mathbb{I}_{\tau}\right)$, we put

$$
\mathfrak{B}(\mathrm{q}(x, \tau))= \begin{cases}\left\{\mathrm{q}\left(\bigcup_{\alpha \leq \tau} U \times\{\alpha\}\right) \mid U \text { is a neighborhood of } 0 \text { in } \mathbb{I}\right\} & \text { if } x=0, \\ \left\{\mathrm{q}\left(\bigcup_{\alpha \leq \tau} U \times\{\alpha\} \backslash \bigcup_{\beta \in \Gamma} \mathbb{I}_{\beta}\right) \mid U\right. \text { is a neighborhood } & \\ \text { of } x \text { in }(0,1], \Gamma \text { is a finite subset of } \tau\} & \text { if } x \neq 0 .\end{cases}
$$


For any other point $\mathrm{q}(x, \alpha)$ in $S^{*}(\tau)$, we let $\mathfrak{B}(\mathrm{q}(x, \alpha))$ be the usual neighborhood base in the hedgehog $S(\tau)$ of spininess $\tau$. Now, we equip $S^{*}(\tau)$ with the topology generated by the neighborhood base $\mathfrak{B}(\mathrm{q}(x, \alpha))$ at every point $\mathrm{q}(x, \alpha)$ in $S^{*}(\tau)$. Note that $S^{*}(\tau)$ can be obtained from the Cartesian product $\mathbb{I} \times \omega D$, where $\omega D$ is the one-point compactification of the discrete space of cardinality $\tau$, be identifying the set $\{0\} \times \omega D$ to a point (this space was considered by Arkhangel'skiı [1, Theorem IV.1.25] and in [5]; cf. [4, Problem 5.3.B(c)]), and $S(\tau)=\mathrm{q}(T(\tau))$ is the usual hedgehog of spininess $\tau$.

Let $\sigma$ be the usual metric on $S(\tau)$. We define the metric $\sigma_{n}$ on the $n$th product of $S(\tau)$ by

$$
\sigma_{n}\left(\left(x_{i}\right),\left(y_{i}\right)\right)=\sum_{i=1}^{n} \frac{\sigma\left(x_{i}, y_{i}\right)}{2^{i}}
$$

for $\left(x_{i}\right)$ and $\left(y_{i}\right)$ in $S(\tau)^{n}$, and the metric $\sigma_{\infty}$ on the countable infinite product of $S(\tau)$ by

$$
\sigma_{\infty}\left(\left(x_{i}\right),\left(y_{i}\right)\right)=\sum_{i=1}^{\infty} \frac{\sigma\left(x_{i}, y_{i}\right)}{2^{i}}
$$

for $\left(x_{i}\right)$ and $\left(y_{i}\right)$ in $S(\tau)^{\mathbb{N}}$.

Let $C(X, Y)$ be the space of all continuous mappings from $X$ into $Y$ endowed with the complete metric defined by

$$
\widetilde{\mathrm{d}}(f, g)=\sup \{\mathrm{d}(f(x), g(x)) \mid x \in X\}
$$

for every pair $(f, g)$ of elements of $C(X, Y)$, where $\mathrm{d}$ is a complete and bounded metric on $Y$.

For a subset $A$ of the $n$th product of $S^{*}(\tau)$, the symbol $\operatorname{cl}_{n} A$ denotes the closure of $A$ in the space, and similarly, for a subset $B$ of the countable infinite product of $S^{*}(\tau)$, the symbol $\mathrm{cl}_{\infty} B$ denotes the closure of $B$ in the space.

Lemma 2.1. Every closed subset $A$ of the countable product of $S^{*}(\tau)$ is Eberlein compact.

Proof. Since being Eberlein compact is preserved by taking countable products, closed subsets and continuous images of such spaces, and the space $S^{*}(\tau)$ is Eberlein compact by the fact mentioned in the definition of the space (cf. also [1, Theorem IV.1.25]), $A$ is Eberlein compact.

Now, we define the subspace $K_{n}^{*}(\tau)$ of $S^{*}(\tau)^{\mathbb{N}}$ for every natural number $n$ as follows:

Definition 2.2. We put

$$
K_{n}^{*}(\tau)=\left\{\left(s_{i}\right) \in S^{*}(\tau)^{\mathbb{N}}||\left\{i \in \mathbb{N} \mid \mathrm{p}\left(s_{i}\right) \in \mathbb{Q} \backslash\{0\}\right\} \mid \leq n\right\} .
$$

LEMMA 2.3. For every compact subset $L$ of $K_{n}^{*}(\tau)$, we have $\operatorname{dim} L \leq n$. 
Proof. Let $\mathbb{Q} \cap(0,1]=\bigcup_{i=1}^{n+1} \mathbb{Q}_{i}$, where $\mathbb{Q}_{i}$ is dense in $\mathbb{I}$ for every $i=$ $1, \ldots, n+1$, and $\mathbb{Q}_{i} \cap \mathbb{Q}_{j}=\emptyset$ for $i \neq j$. For every $i=1, \ldots, n+1$, we put

$$
\begin{aligned}
P_{i} & =\left\{\left(s_{j}\right) \in K_{n}^{*}(\tau) \mid \mathrm{p}\left(s_{j}\right) \in \mathbb{Q}_{i} \text { for some } j \in \mathbb{N}\right\}, \\
\mathfrak{B}_{i} & =\left\{U \mid U \text { is open in } K_{n}^{*}(\tau) \text { and } \operatorname{Bd} U \subset P_{i}\right\} .
\end{aligned}
$$

Notice that $\mathfrak{B}_{i}$ is a base for $K_{n}^{*}(\tau)$ for every $i$. Since $L$ is compact, for every pair $\left(E_{i}, F_{i}\right)$ of disjoint closed subsets of $L(i=1, \ldots, n+1)$, there exists a finite subset $\mathfrak{B}_{i}^{\prime}$ of $\mathfrak{B}_{i}$ such that

$$
E_{i} \subset\left(\bigcup \mathfrak{B}_{i}^{\prime}\right) \cap L \subset \operatorname{cl}_{\infty}\left(\left(\bigcup \mathfrak{B}_{i}^{\prime}\right) \cap L\right) \subset L \backslash F_{i} .
$$

Let $L_{i}=\operatorname{Bd}\left(\bigcup \mathfrak{B}_{i}^{\prime}\right)$. Then $L_{i}$ is a partition of $L$ between $E_{i}$ and $F_{i}$. Since $\bigcap_{i=1}^{n+1} L_{i}=\emptyset$, we have $\operatorname{dim} L \leq n$.

Now, we define

$$
\mathfrak{K}=((0,1] \cap \mathbb{Q})^{n+1}, \quad \mathfrak{J}=\left\{\left(i_{1}, \ldots, i_{n+1}\right) \mid i_{j} \in \mathbb{N}, \text { and } i_{j} \neq i_{k} \text { for } j \neq k\right\} .
$$

For $K=\left(q_{1}, \ldots, q_{n+1}\right) \in \mathfrak{K}$ and $J=\left(i_{1}, \ldots, i_{n+1}\right) \in \mathfrak{J}$, let

$$
F(K, J)=\left\{\left(s_{i}\right) \in S^{*}(\tau)^{\mathbb{N}} \mid \mathrm{p}\left(s_{i_{j}}\right)=q_{j} \text { for } j=1, \ldots, n+1\right\} .
$$

LEMMA 2.4. For every closed subset $A$ of $S(\tau)^{\mathbb{N}}$, the following are equivalent:

(1) $\mathrm{cl}_{\infty} A \cap F(K, J)=\emptyset$,

(2) $\sigma_{\infty}\left(A, F(K, J) \cap S(\tau)^{\mathbb{N}}\right)>0$.

Proof. First, we prove $(2) \Rightarrow(1)$. Suppose not, and take a point $s=\left(s_{i}\right)$ $\in \mathrm{cl}_{\infty} A \cap F(K, J)$. Put $\delta=\sigma_{\infty}\left(A, F(K, J) \cap S(\tau)^{\mathbb{N}}\right)$. Take $k$ such that $1 / 2^{k}<\delta / 8$. Since $s \in \operatorname{cl}_{\infty} A$, there exists a point $t=\left(t_{i}\right) \in A$ such that

$$
\begin{cases}\lambda\left(s_{i}\right)=\lambda\left(t_{i}\right) & \text { if } \lambda\left(s_{i}\right) \neq \tau \\ \left|\mathrm{p}\left(s_{i}\right)-\mathrm{p}\left(t_{i}\right)\right|<\delta / 4 & \text { for every } i\end{cases}
$$

We put

$$
u_{i}= \begin{cases}s_{i} & \text { if } \lambda\left(s_{i}\right) \neq \tau \\ \mathrm{q}\left(\mathrm{p}\left(s_{i}\right), \lambda\left(t_{i}\right)\right) & \text { if } \lambda\left(s_{i}\right)=\tau .\end{cases}
$$

We see that $u=\left(u_{i}\right) \in F(K, J) \cap S(\tau)^{\mathbb{N}}$. We have

$$
\begin{aligned}
\sigma_{\infty}(t, u) & =\sum_{i=1}^{\infty} \frac{\sigma\left(t_{i}, u_{i}\right)}{2^{i}} \leq \sum_{i=1}^{k} \frac{\sigma\left(t_{i}, u_{i}\right)}{2^{i}}+\sum_{i=k+1}^{\infty} \frac{\operatorname{diam}_{\sigma} S(\tau)}{2^{i}} \\
& \leq \frac{\delta}{4}+\frac{\delta}{8} \cdot 2=\frac{\delta}{2} .
\end{aligned}
$$

This contradicts $\sigma_{\infty}\left(A, F(K, J) \cap S(\tau)^{\mathbb{N}}\right)=\delta$. 
Now, we prove $(1) \Rightarrow(2)$. By Wallace's theorem (see [3, Theorem 3.2.10]), for every $j=1, \ldots, n+1$, there exists an open subset $U_{j}$ of $S^{*}(\tau)$ such that

$$
F(K, J) \subset \bigcap_{j=1}^{n+1} \pi_{i_{j}}^{-1}\left(U_{j}\right) \subset \operatorname{cl}_{\infty}\left(\bigcap_{j=1}^{n+1} \pi_{i_{j}}^{-1}\left(U_{j}\right)\right) \subset S^{*}(\tau)^{\mathbb{N}} \backslash \mathrm{cl}_{\infty} A,
$$

where $\pi_{l}$ is the $l$ th projection from $S^{*}(\tau)^{\mathbb{N}}$ into $S^{*}(\tau)$. By the definition of $F(K, J)$, we can find a positive number $\varepsilon_{j}$ such that

$$
\mathrm{q}\left(\left(q_{j}-\varepsilon_{j}, q_{j}+\varepsilon_{j}\right) \times \lambda\left(s_{i_{j}}\right)\right) \subset U_{j}
$$

for every $j$. Put

$$
\varepsilon=\min \left\{\varepsilon_{j} \mid 1 \leq j \leq n+1\right\}, \quad l=\max \left\{i_{j} \mid 1 \leq j \leq n+1\right\} .
$$

We have

$$
\varepsilon / 2^{l} \leq \sigma_{\infty}\left(A, F(K, J) \cap S(\tau)^{\mathbb{N}}\right)
$$

Recall that a subset of a space $X$ is said to be residual if it contains a dense $G_{\delta^{-}}$-subset of $X$. The proof of Lemma 2.5 is essentially due to Pol [9].

Lemma 2.5. Let $X$ be a normal space and $A$ a closed subset of $X$ with $\operatorname{dim} A \leq n$. For every cardinal $\tau$, the set $\mathcal{H}=\left\{h \in C\left(X, S(\tau)^{\mathbb{N}}\right) \mid\right.$ $\left.\mathrm{cl}_{\infty} h(A) \subset K_{n}^{*}(\tau)\right\}$ is residual in $C\left(X, S(\tau)^{\mathbb{N}}\right)$.

Proof. For every $K=\left(q_{1}, \ldots, q_{n+1}\right) \in \mathfrak{K}$ and $J=\left(i_{1}, \ldots, i_{n+1}\right) \in \mathfrak{J}$, we put

$$
\mathcal{F}(K, J)=\left\{f \in C\left(X, S(\tau)^{\mathbb{N}}\right) \mid \operatorname{cl}_{\infty} f(A) \cap F(K, J)=\emptyset\right\} .
$$

Since

$$
S^{*}(\tau)^{\mathbb{N}} \backslash K_{n}^{*}(\tau)=\bigcup\{F(K, J) \mid K \in \mathfrak{K}, J \in \mathfrak{J}\},
$$

we have

$$
\bigcap\{\mathcal{F}(K, J) \mid K \in \mathfrak{K}, J \in \mathfrak{J}\} \subset \mathcal{H} .
$$

By Lemma 2.4, we can see that $\mathcal{F}(K, J)$ is an open subset of $C\left(X, S(\tau)^{\mathbb{N}}\right)$. Hence, it is sufficient to prove that $\mathcal{F}(K, J)$ is a dense subset of $C\left(X, S(\tau)^{\mathbb{N}}\right)$. Take an arbitrary mapping $f=\left(f_{i}\right): X \rightarrow S(\tau)^{\mathbb{N}}$ and $\varepsilon$. For every $i \in \mathbb{N}$ with $i \neq i_{j}$, we put $g_{i}=f_{i}$. Set

$$
F=\left(\prod_{j=1}^{n+1} \mathrm{p}^{-1}\left(q_{j}\right)\right) \cap S(\tau)^{n+1}
$$

Below, we show that, for every $i_{j}$, there exists a mapping $g_{i_{j}}$ in $C(X, S(\tau))$ such that $\sigma\left(g_{i_{j}}(x), f_{i_{j}}(x)\right)<\varepsilon$ for every $x \in X$, and $\sigma_{n+1}(\bar{g}(A), F)>0$ for $\bar{g}=\left(g_{i_{j}}\right): X \rightarrow S(\tau)^{n+1}$. Then it is obvious that the mapping $g=\left(g_{i}\right)$ from $X$ into $S(\tau)^{\mathbb{N}}$ satisfies

$$
\operatorname{cl}_{\infty} g(A) \cap F(K, J)=\emptyset \quad \text { and } \quad \tilde{\sigma}_{\infty}(f, g)<\varepsilon .
$$


Put $T=\tau^{n+1}$. For every $t=\left(\alpha_{1}, \ldots, \alpha_{n+1}\right) \in T$, we put $p_{t}=$ $\left(\mathrm{q}\left(q_{j}, \alpha_{j}\right)\right)_{j=1}^{n+1}$. Notice that $F=\left\{p_{t} \mid t \in T\right\}$. Take $\eta$ with

$$
0<\eta<\min \left\{q_{1}, \ldots, q_{n+1}, \varepsilon / 2\right\} .
$$

For every $t=\left(\alpha_{1}, \ldots, \alpha_{n+1}\right) \in T$, we put

$$
\begin{aligned}
U_{t} & =\left\{\left(s_{i}\right) \in S(\tau)^{n+1}\left|\lambda\left(s_{i}\right)=\alpha_{i},\right| \mathrm{p}\left(s_{i}\right)-q_{i} \mid<\eta\right\}, \\
K_{t} & =\operatorname{cl}_{n+1} U_{t}, \quad S_{t}=K_{t} \backslash U_{t} .
\end{aligned}
$$

Write $\bar{f}=\left(f_{i_{j}}\right)$. Then $\left\{U_{t} \mid t \in T\right\}$ is a discrete collection of open neighborhoods of the points $p_{t}$ in $S(\tau)^{n+1}$ and the family

$$
\left\{\bar{f}^{-1}\left(K_{t}\right) \mid t \in T\right\} \cup\left\{\bar{f}^{-1}\left(S(\tau)^{n+1} \backslash \bigcup_{t \in T} U_{t}\right)\right\}
$$

is a locally finite closed covering of $X$. We consider two cases.

CASE $1: q_{i}+\eta \leq 1$ for every $i=1, \ldots, n+1$. For every $t \in T$, the set $S_{t}$ is homeomorphic to the $n$-dimensional sphere, and $\operatorname{since} \operatorname{dim} A \leq n$, there exists a mapping $h_{t}: \bar{f}^{-1}\left(K_{t}\right) \cap A \rightarrow S_{t}$ such that $\left.h_{t}\right|_{\bar{f}^{-1}\left(S_{t}\right) \cap A}=$ $\left.\bar{f}\right|_{\bar{f}^{-1}\left(S_{t}\right) \cap A}$. We define $h_{t}^{\prime}:\left(\bar{f}^{-1}\left(K_{t}\right) \cap A\right) \cup \bar{f}^{-1}\left(S_{t}\right) \rightarrow S_{t}$ by

$$
h_{t}^{\prime}(x)= \begin{cases}h_{t}(x) & \text { if } x \in \bar{f}^{-1}\left(K_{t}\right) \cap A, \\ \bar{f}(x) & \text { otherwise. }\end{cases}
$$

Since $\left(\bar{f}^{-1}\left(K_{t}\right) \cap A\right) \cup \bar{f}^{-1}\left(S_{t}\right)$ is a closed subset of the normal space $X$, there exists a continuous mapping $g_{t}: \bar{f}^{-1}\left(K_{t}\right) \rightarrow K_{t}$ such that

$$
\left.g_{t}\right|_{\bar{f}^{-1}\left(S_{t}\right)}=\left.\bar{f}\right|_{\bar{f}^{-1}\left(S_{t}\right)},\left.\quad g_{t}\right|_{A \cap \bar{f}^{-1}\left(K_{t}\right)}=h_{t} .
$$

We have $\sigma_{n+1}\left(g_{t}\left(A \cap \bar{f}^{-1}\left(K_{t}\right)\right), p_{t}\right) \geq \eta$. Now, we put $g^{\prime}=\left.\bar{f}\right|_{\bar{f}^{-1}\left(S(\tau)^{n+1} \backslash \cup_{t \in T} U_{t}\right)}$. Let $\bar{g}: X \rightarrow S(\tau)^{n+1}$ be the combination of $g^{\prime}$ and the mappings $\left\{g_{t} \mid t \in T\right\}$. Then $\bar{g}$ is continuous. Moreover,

$$
\sigma_{n+1}(\bar{g}(A), F) \geq \eta, \quad \sigma\left(f_{i_{j}}(x), g_{i_{j}}(x)\right) \leq 2 \eta<\varepsilon \quad \text { for every } x \in X .
$$

CASE 2: $q_{i}+\eta>1$ for some $i=1, \ldots, n+1$. Then there exists a continuous retraction $r_{t}: K_{t} \rightarrow S_{t}$. Set $g_{t}=\left.r_{t} \circ \bar{f}\right|_{\bar{f}^{-1}\left(K_{t}\right)}$. We define the mappings $g^{\prime}$ and $\bar{g}$ as in Case 1 and we reach the same conclusion.

Below, the symbol $\mathcal{E}(M)$ denotes the set

$$
\left\{h \in C\left(M, S(\tau)^{\mathbb{N}}\right) \mid h \text { is a homeomorphic embedding }\right\}
$$

for a metrizable space $M$ with $w(M)=\tau$.

Lemma 2.6 (Toruńczyk, see [9]). If $M$ is a metrizable space with $w(M)=\tau$, then the set $\mathcal{E}(M)$ is residual in $C\left(M, S(\tau)^{\mathbb{N}}\right)$.

By Lemmas 2.3, 2.5 and 2.6, we have the following theorem. 
TheOREM 2.7. Let $M$ be a metrizable space with $w(M)=\tau$ and $\operatorname{dim} M=n$. The set

$$
\left\{h \in \mathcal{E}(M) \mid \operatorname{dim} \mathrm{cl}_{\infty} h(M) \leq n\right\}
$$

is residual in $C\left(M, S(\tau)^{\mathbb{N}}\right)$.

Now, we proceed to prove the residuality of $\left\{h \in \mathcal{E}(M) \mid \operatorname{dim}_{\infty} h(M)\right.$ $\geq n\}$. To do this, we need some lemmas. Lemma 2.10 will be used again in the next section. Below, for a positive number $\varepsilon$, a positive integer $n$ and a subset $Y$ of $S(\tau)^{n}, N(Y, \varepsilon, n)$ denotes the $\varepsilon$-neighborhood of $Y$ in $S(\tau)^{n}$, and similarly, for a subset $Z$ of $S(\tau)^{\mathbb{N}}, N(Z, \varepsilon, \infty)$ denotes the $\varepsilon$-neighborhood of $Z$ in $S(\tau)^{\mathbb{N}}$. For subsets $A$ and $B$ of $S(\tau)^{n}$ with $\operatorname{cl}_{n} A \cap \operatorname{cl}_{n} B=\emptyset$, a positive number $\varepsilon$ is said to be a separating constant of $(A, B)$ if $\operatorname{cl}_{n} N(A, \varepsilon, n) \cap$ $\operatorname{cl}_{n} N(B, \varepsilon, n)=\emptyset$.

Lemma 2.8. If $A, B$ are subsets of $S(\tau)^{n}$ with $\operatorname{cl}_{n} A \cap \operatorname{cl}_{n} B=\emptyset$, then there exists a separating constant of $(A, B)$.

Proof. For simplicity, we give the proof in the case of $n=2$. Take subsets $A$ and $B$ of $S(\tau)^{2}$ with $\operatorname{cl}_{2} A \cap \mathrm{cl}_{2} B=\emptyset$. For every pair $(\alpha, \beta)$ in $(\tau+1)^{2}$, we put

$$
A_{(\alpha, \beta)}=\left(\mathrm{q}\left(\mathbb{I}_{\alpha}\right) \times \mathrm{q}\left(\mathbb{I}_{\beta}\right)\right) \cap \mathrm{cl}_{2} A, \quad B_{(\alpha, \beta)}=\left(\mathrm{q}\left(\mathbb{I}_{\alpha}\right) \times \mathrm{q}\left(\mathbb{I}_{\beta}\right)\right) \cap \mathrm{cl}_{2} B .
$$

Let $\mathrm{p}_{2}: S^{*}(\tau)^{2} \rightarrow \mathbb{I}^{2}$ be defined by $\mathrm{p}_{2}\left(\mathrm{q}\left(x_{1}, a_{1}\right), \mathrm{q}\left(x_{2}, a_{2}\right)\right)=\left(x_{1}, x_{2}\right)$. We can find open subsets $U_{(\alpha, \beta)}$ and $V_{(\alpha, \beta)}$ of $\mathbb{I}^{2}$ with

$$
\mathrm{p}_{2}\left(A_{(\alpha, \beta)}\right) \subset U_{(\alpha, \beta)}, \quad \mathrm{p}_{2}\left(B_{(\alpha, \beta)}\right) \subset V_{(\alpha, \beta)}, \quad \mathrm{cl}_{\mathbb{I}^{2}} U_{(\alpha, \beta)} \cap \mathrm{cl}_{\mathbb{I}^{2}} V_{(\alpha, \beta)}=\emptyset
$$

for every pair $(\alpha, \beta)$, and a finite subset $\Lambda$ of $\tau$ with

$$
\mathrm{p}_{2}\left(A_{(\alpha, \beta)}\right) \subset U_{(\tau, \tau)}, \quad \mathrm{p}_{2}\left(B_{(\alpha, \beta)}\right) \subset V_{(\tau, \tau)}
$$

for every pair $(\alpha, \beta)$ in $(\tau \backslash \Lambda)^{2}$. Put

$$
\begin{aligned}
& A_{1}=\bigcup\left\{A_{(\alpha, \beta)} \backslash \mathrm{p}_{2}^{-1}\left(U_{(\tau, \tau)}\right) \mid \alpha \notin \Lambda \text { and } \beta<\tau+1\right\} \text {, } \\
& A_{2}=\bigcup\left\{A_{(\alpha, \beta)} \backslash \mathrm{p}_{2}^{-1}\left(U_{(\tau, \tau)}\right) \mid \alpha<\tau+1 \text { and } \beta \notin \Lambda\right\} \text {, } \\
& B_{1}=\bigcup\left\{B_{(\alpha, \beta)} \backslash \mathrm{p}_{2}^{-1}\left(V_{(\tau, \tau)}\right) \mid \alpha \notin \Lambda \text { and } \beta<\tau+1\right\} \text {, } \\
& B_{2}=\bigcup\left\{B_{(\alpha, \beta)} \backslash \mathrm{p}_{2}^{-1}\left(V_{(\tau, \tau)}\right) \mid \alpha<\tau+1 \text { and } \beta \notin \Lambda\right\} \text {, } \\
& F_{1}=\mathbb{I} \times\{0\}, \quad F_{2}=\{0\} \times \mathbb{I} .
\end{aligned}
$$

We have

$$
\mathrm{p}_{2}\left(A_{1} \cup B_{1}\right) \cap F_{1}=\emptyset, \quad \mathrm{p}_{2}\left(A_{2} \cup B_{2}\right) \cap F_{2}=\emptyset .
$$

Let $\varrho$ be the metric on $\mathbb{I}^{2}$ defined by

$$
\varrho\left(\left(x_{1}, x_{2}\right),\left(y_{1}, y_{2}\right)\right)=\frac{1}{2}\left|x_{1}-y_{1}\right|+\frac{1}{4}\left|x_{2}-y_{2}\right|
$$


for $\left(x_{1}, x_{2}\right),\left(y_{1}, y_{2}\right) \in \mathbb{I}^{2}$ (for general $n$, the metric $\varrho$ on $\mathbb{I}^{n}$ should be defined by $\left.\varrho\left(\left(x_{i}\right),\left(y_{i}\right)\right)=\sum_{i=1}^{n}\left|x_{i}-y_{i}\right| / 2^{i}\right)$. Put

$$
\varepsilon_{1}=\min \left\{\varrho\left(\mathrm{cl}_{\mathbb{I}^{2}} U_{(\tau, \tau)}, \mathrm{cl}_{\mathbb{I}^{2}} V_{(\tau, \tau)}\right), \varrho\left(\mathrm{p}_{2}\left(A_{1} \cup B_{1}\right), F_{1}\right), \varrho\left(\mathrm{p}_{2}\left(A_{2} \cup B_{2}\right), F_{2}\right)\right\} .
$$

For every $\alpha$ in $\Lambda$, there exists a finite subset $\Lambda_{\alpha}$ of $\tau$ such that

$$
\begin{array}{ll}
\mathrm{p}_{2}\left(A_{(\alpha, \beta)}\right) \subset U_{(\alpha, \tau)}, & \mathrm{p}_{2}\left(A_{(\beta, \alpha)}\right) \subset U_{(\tau, \alpha)}, \\
\mathrm{p}_{2}\left(B_{(\alpha, \beta)}\right) \subset V_{(\alpha, \tau)}, & \mathrm{p}_{2}\left(B_{(\beta, \alpha)}\right) \subset V_{(\tau, \alpha)},
\end{array}
$$

for every $\beta$ in $\tau \backslash \Lambda_{\alpha}$. For every $\alpha$ in $\Lambda$, we put

$$
\begin{aligned}
A(\alpha) & =\bigcup\left\{A_{(\alpha, \beta)} \backslash \mathrm{p}_{2}^{-1}\left(U_{(\alpha, \tau)}\right) \mid \beta<\tau+1, \beta \in \Lambda_{\alpha}\right\}, \\
A^{\prime}(\alpha) & =\bigcup\left\{A_{(\beta, \alpha)} \backslash \mathrm{p}_{2}^{-1}\left(U_{(\tau, \alpha)}\right) \mid \beta<\tau+1, \beta \in \Lambda_{\alpha}\right\}, \\
B(\alpha) & =\bigcup\left\{B_{(\alpha, \beta)} \backslash \mathrm{p}_{2}^{-1}\left(V_{(\alpha, \tau)}\right) \mid \beta<\tau+1, \beta \in \Lambda_{\alpha}\right\}, \\
B^{\prime}(\alpha) & =\bigcup\left\{B_{(\beta, \alpha)} \backslash \mathrm{p}_{2}^{-1}\left(V_{(\tau, \alpha)}\right) \mid \beta<\tau+1, \beta \in \Lambda_{\alpha}\right\} .
\end{aligned}
$$

We have

$$
\mathrm{p}_{2}(A(\alpha) \cup B(\alpha)) \cap F_{1}=\emptyset, \quad \mathrm{p}_{2}\left(A^{\prime}(\alpha) \cup B^{\prime}(\alpha)\right) \cap F_{2}=\emptyset .
$$

Put

$$
\begin{aligned}
\delta_{\alpha}=\min \left\{\varrho\left(\mathrm{cl}_{\mathbb{I}^{2}} U_{(\alpha, \tau)}, \mathrm{cl}_{\mathbb{I}^{2}} V_{(\alpha, \tau)}\right), \varrho\left(\mathrm{cl}_{\mathbb{I}^{2}} U_{(\tau, \alpha)}, \mathrm{cl}_{\mathbb{I}^{2}} V_{(\tau, \alpha)}\right),\right. \\
\left.\varrho\left(\mathrm{p}_{2}(A(\alpha) \cup B(\alpha)), F_{1}\right), \varrho\left(\mathrm{p}_{2}\left(A^{\prime}(\alpha) \cup B^{\prime}(\alpha)\right), F_{2}\right)\right\}
\end{aligned}
$$

for every $\alpha$ in $\Lambda$, and

$$
\delta_{(\alpha, \beta)}=\varrho\left(\mathrm{p}_{2}\left(A_{(\alpha, \beta)}\right), \mathrm{p}_{2}\left(B_{(\alpha, \beta)}\right)\right)
$$

for every pair $(\alpha, \beta)$. Finally, we put

$$
\begin{aligned}
\varepsilon_{2} & =\min \left\{\delta_{\alpha} \mid \alpha \in \Lambda\right\}, \\
\varepsilon_{3} & =\min \left\{\delta_{(\alpha, \beta)} \mid \alpha \in \Lambda, \beta \in \Lambda_{\alpha}\right\}, \\
\varepsilon_{4} & =\min \left\{\delta_{(\beta, \alpha)} \mid \alpha \in \Lambda, \beta \in \Lambda_{\alpha}\right\}, \\
\varepsilon & =\frac{1}{3} \min \left\{\varepsilon_{1}, \varepsilon_{2}, \varepsilon_{3}, \varepsilon_{4}\right\} .
\end{aligned}
$$

This $\varepsilon$ is as desired.

Lemma 2.9. Let $X_{k}$ be a compact space for every $k \in \mathbb{N}, A$ and $B$ closed subsets of $\prod_{k \in \mathbb{N}} X_{k}$, and $\Pi_{n}: \prod_{k \in \mathbb{N}} X_{k} \rightarrow \prod_{1 \leq k \leq n} X_{k}$ the projection map. If $A \cap B=\emptyset$ then there exists a positive integer $n$ such that $\Pi_{n}(A) \cap \Pi_{n}(B)=\emptyset$.

Proof. Suppose that there exist points $a_{n}$ in $A$ and $b_{n}$ in $B$ such that $\Pi_{n}\left(a_{n}\right)=\Pi_{n}\left(b_{n}\right)$ for every $n$. Let $a$ be a cluster point of the set $\left\{a_{n} \mid n \in \mathbb{N}\right\}$. We can easily show that $a$ belongs to $B$. 
Lemma 2.10. Let $M$ be a metrizable space and $A$ and $B$ disjoint closed subsets of $M$. The set

$$
\mathcal{S}=\left\{h \in C\left(M, S(\tau)^{\mathbb{N}}\right) \mid \operatorname{cl}_{\infty} h(A) \cap \operatorname{cl}_{\infty} h(B)=\emptyset\right\}
$$

is open and dense in $C\left(M, S(\tau)^{\mathbb{N}}\right)$.

Proof. First, we show that $\mathcal{S}$ is a dense subset of $C\left(M, S(\tau)^{\mathbb{N}}\right)$. Take any $f=\left(f_{i}\right) \in C\left(M, S(\tau)^{\mathbb{N}}\right)$ and $\varepsilon>0$. Let $k$ be a positive integer with $1 / 2^{k}<\varepsilon$. Put

$$
g_{i}= \begin{cases}f_{i} & (i \neq k), \\ \psi & (i=k),\end{cases}
$$

where $\psi: M \rightarrow S(\tau)$ with $\psi(A) \subseteq\{q(0,0)\}$ and $\psi(B) \subseteq\{q(1,0)\}$. Then it is obvious that $g=\left(g_{i}\right): M \rightarrow S(\tau)^{\mathbb{N}}$ satisfies $g \in \mathcal{S}$ and $\widetilde{\sigma}_{\infty}(f, g)<\varepsilon$.

Next, we show that $\mathcal{S}$ is an open subset of $C\left(M, S(\tau)^{\mathbb{N}}\right)$. Take $h \in \mathcal{S}$. By Lemma 2.9, there exists a positive integer $n$ such that $\Pi_{n}\left(\operatorname{cl}_{\infty} h(A)\right) \cap$ $\Pi_{n}\left(\mathrm{cl}_{\infty} h(B)\right)=\emptyset$. Since $\mathrm{cl}_{n} \Pi_{n}(h(A)) \cap \mathrm{cl}_{n} \Pi_{n}(h(B))=\emptyset$, by Lemma 2.8, there exists a separating constant $\varepsilon$ of $\left(\Pi_{n}(h(A)), \Pi_{n}(h(B))\right)$. Let $h^{\prime} \in$ $C\left(M, S(\tau)^{\mathbb{N}}\right)$ satisfy $\widetilde{\sigma}_{\infty}\left(h, h^{\prime}\right)<\varepsilon$. We have

$$
h^{\prime}(A) \subset N(h(A), \varepsilon, \infty), \quad h^{\prime}(B) \subset N(h(B), \varepsilon, \infty) .
$$

Since

$$
\Pi_{n}\left(h^{\prime}(A)\right) \subset N\left(\Pi_{n}(h(A)), \varepsilon, n\right), \quad \Pi_{n}\left(h^{\prime}(B)\right) \subset N\left(\Pi_{n}(h(B)), \varepsilon, n\right),
$$

and $\varepsilon$ is a separating constant of $\left(\Pi_{n}(h(A)), \Pi_{n}(h(B))\right.$, we have

$$
\mathrm{cl}_{n} \Pi_{n}\left(h^{\prime}(A)\right) \cap \mathrm{cl}_{n} \Pi_{n}\left(h^{\prime}(B)\right)=\emptyset .
$$

This implies

$$
\mathrm{cl}_{\infty} h^{\prime}(A) \cap \mathrm{cl}_{\infty} h^{\prime}(B)=\emptyset .
$$

Recall that a family $\left\{\left(A_{i}, B_{i}\right) \mid 1 \leq i \leq n\right\}$ of pairs of disjoint closed subsets of a space is said to be essential if, for every $i$ and every partition $L_{i}$ between $A_{i}$ and $B_{i}, \bigcap_{i=1}^{n} L_{i} \neq \emptyset$.

THEOREM 2.11. Let $M$ be a metrizable space with $w(M)=\tau$ and $\operatorname{dim} M$ $=n \in \mathbb{N}$. The set

$$
\left\{h \in \mathcal{E}(M) \mid \operatorname{dimcl}_{\infty} h(M) \geq n\right\}
$$

is residual in $C\left(M, S(\tau)^{\mathbb{N}}\right)$.

Proof. Since $\operatorname{dim} M=n$, there exists an essential family $\left\{\left(A_{i}, B_{i}\right)\right.$ $1 \leq i \leq n\}$ of pairs of closed subsets of $M$. By Lemmas 2.6 and 2.10, the set

$$
\left\{h \in \mathcal{E}(M) \mid \mathrm{cl}_{\infty} h\left(A_{i}\right) \cap \mathrm{cl}_{\infty} h\left(B_{i}\right)=\emptyset \text { for every } 1 \leq i \leq n\right\}
$$

is residual in $C\left(M, S(\tau)^{\mathbb{N}}\right)$. For every $h$ in this set, the set

$$
\left\{\left(\operatorname{cl}_{\infty} h\left(A_{i}\right), \mathrm{cl}_{\infty} h\left(B_{i}\right)\right) \mid 1 \leq i \leq n\right\}
$$

is essential. Therefore, $\operatorname{dim} \mathrm{cl}_{\infty} h(M) \geq n$. 
Theorems 2.7, 2.11 and Lemma 2.1 yield the assertion of Theorem 1.1.

Now, we investigate the case of infinite-dimensional spaces.

Lemma 2.12 ([4, Problem 6.1.E(c), p. 310]). A weakly paracompact normal space $X$ is $S$-w.i.d. if and only if $X$ contains an $S$-w.i.d. compact subset $L$ such that each closed subset of $X$ disjoint from $L$ is finite-dimensional.

Notice that compact spaces and metrizable spaces are weakly paracompact.

Theorem 2.13. Let $M$ be an S-w.i.d. metrizable space with $w(M)=\tau$. The set

$$
\left\{h \in \mathcal{E}(M) \mid \operatorname{cl}_{\infty} h(M) \text { is S-w.i.d. }\right\}
$$

is residual in $C\left(M, S(\tau)^{\mathbb{N}}\right)$.

Proof. Let $L$ be a compact subset of $M$ as in Lemma 2.12. Put

$$
F_{n}=\{x \in M \mid \mathrm{d}(x, L) \geq 1 / n\}
$$

for every $n \in \mathbb{N}$, where $\mathrm{d}$ is a metric on $M$ that is compatible with its topology. The dimension of the closed subset $F_{n}$ is finite. We put $l(n)=$ $\operatorname{dim} F_{n}$. Set

$$
\begin{aligned}
\mathcal{H}_{n} & =\left\{h \in C\left(M, S(\tau)^{\mathbb{N}}\right) \mid \operatorname{cl}_{\infty} h\left(F_{n}\right) \subset K_{l(n)}^{*}(\tau)\right\}, \\
\mathcal{H} & =\bigcap\left\{\mathcal{H}_{n} \mid n \in \mathbb{N}\right\} \cap \mathcal{E}(M) .
\end{aligned}
$$

The set $\mathcal{H}$ is residual in $C\left(M, S(\tau)^{\mathbb{N}}\right)$ by Lemmas 2.5 and 2.6. Take an embedding $h$ in $\mathcal{H}$. Notice that $h(L)$ is an S-w.i.d. compact subset of $\operatorname{cl}_{\infty} h(M)$. By Lemma 2.12, it is sufficient to show that each closed subset $A$ of $\operatorname{cl}_{\infty} h(M)$ that is disjoint from $h(L)$ is finite-dimensional. Since $h(L)$ is compact, there exists an open subset $U$ of $\operatorname{cl}_{\infty} h(M)$ such that $A \subset U$ and $\operatorname{cl}_{\infty} U \cap h(L)=\emptyset$. Put $B=h^{-1}\left(\operatorname{cl}_{\infty} U \cap h(M)\right)$. Since $B$ is a closed subset of $M$ that is disjoint from the compact subset $L$, there exists $k$ such that $B \subset F_{k}$. By Lemma 2.3, $\operatorname{dim} \operatorname{cl}_{\infty} h\left(F_{k}\right) \leq l(k)$. Since $A$ is a closed subset of $\operatorname{cl}_{\infty} h\left(F_{k}\right)$, $A$ is finite-dimensional.

Theorem 2.13 and Lemma 2.1 give the assertion of Theorem 1.2.

Theorem 2.13 is a counterpart for non-separable spaces of Corollary 4.4 of [8]. Using Lemma 2.12, we can prove the following counterpart of Corollary 4.3 of [8].

THEOREM 2.14. Let $M$ be a metrizable space with $w(M)=\tau$ which has the large transfinite dimension trInd. The set

$$
\left\{h \in \mathcal{E}(M) \mid \mathrm{cl}_{\infty} h(M) \text { is countable-dimensional }\right\}
$$

is residual in $C\left(M, S(\tau)^{\mathbb{N}}\right)$. 
Corollary 2.15. Let $M$ be a metrizable space having trInd. There exists a countable-dimensional Eberlein compactification $E$ of $M$ with $w(E)$ $=w(M)$.

REMARK 2.16. It is known that there exists a countable-dimensional metrizable space which has no countable-dimensional compactification (see [6]). The proof of Proposition 2 of [5] yields in fact the statement: Each countable-dimensional complete metrizable space can be embedded in a countable-dimensional Eberlein compact space of the same weight.

3. Related topics. In this section, we mention some results that are related to Lemma 2.10 of Section 2. We use the same terminology as in Section 2. First, we have

Proposition 3.1. Let $M$ be a metrizable space with $w(M)=\tau$ and $f$ a continuous mapping from $M$ into a compact metrizable space $Y$. The set $\left\{h \in \mathcal{E}(M) \mid\right.$ there exists a mapping $\widehat{f}$ in $C\left(\mathrm{cl}_{\infty} h(M), Y\right)$ such that $\widehat{f} \circ h=f\}$

is residual in $C\left(M, S(\tau)^{\mathbb{N}}\right)$.

Proof. Let $\mathcal{B}$ be a countable base for $Y$ that is closed under taking finite unions. Let $\mathcal{A}$ be the family of pairs of disjoint closures of elements of $\mathcal{B}$. Since $\mathcal{A}$ is countable, the set

$$
\left\{h \in \mathcal{E}(M) \mid \operatorname{cl}_{\infty} h\left(f^{-1}(A)\right) \cap \mathrm{cl}_{\infty} h\left(f^{-1}(B)\right)=\emptyset \text { for every }(A, B) \in \mathcal{A}\right\}
$$

is residual in $C\left(M, S(\tau)^{\mathbb{N}}\right)$ by Lemmas 2.6 and 2.10. For every $h$ in the set, there exists a continuous mapping $\widehat{f}: \operatorname{cl}_{\infty} h(M) \rightarrow Y$ such that $\widehat{f} \circ h=f$ by Theorem 3.2.1 of [3].

Combining Proposition 3.1 with Theorems 2.7, 2.11 and Lemma 2.1, we have

THEOREM 3.2. Let $M$ be a finite-dimensional metrizable space and $\left(f_{n}\right)$ a sequence of continuous mappings from $M$ into compact metrizable spaces. There exists an Eberlein compactification $E$ of $M$ such that $\operatorname{dim} E=$ $\operatorname{dim} M, w(E)=w(M)$ and every $f_{n}$ has a continuous extension over $E$.

This theorem is a counterpart for non-separable spaces of Theorem 3.4 of [7].

Similarly, we have

Theorem 3.3. Let $M$ be an $S$-w.i.d. metrizable space and $\left(f_{n}\right)$ a sequence of continuous mappings from $M$ into compact metrizable spaces. There exists an $S$-w.i.d. Eberlein compactification $E$ of $M$ such that $w(E)=$ $w(M)$ and every $f_{n}$ has a continuous extension over $E$. 
We conclude the paper with a result on the Stone-Čech compactification of a metrizable space.

Proposition 3.4. Let $M$ be a metrizable space. The Stone- $\check{C}$ ech compactification $\beta M$ of $M$ is the supremum of the set of all Eberlein compactifications of $M$.

Proof. By Lemmas 2.10 and 2.1, for every pair $(A, B)$ of disjoint closed subsets of $M$, we can find an Eberlein compactification $E$ of $M$ such that the closures of $A$ and $B$ in $E$ are disjoint. Therefore, $\beta M$ is the supremum of such compactifications.

REMARK 3.5. It is known that if the Stone-Cech compactification of a space $X$ is Eberlein compact, so is $X$ (see [1]).

Remark 3.6. Proposition 3.4 can be extended to the class of all spaces (not necessarily metrizable) that have Eberlein compactifications.

\section{References}

[1] A. V. Arhangel'skiı̌, Topological Function Spaces, Kluwer, 1992.

[2] G. Dimov, On Eberlein spaces and related spaces, C. R. Acad. Sci. Paris Sér. I 304 (1987), 233-235.

[3] R. Engelking, General Topology, Heldermann, 1989.

[4] —, Theory of Dimensions, Finite and Infinite, Heldermann, 1995.

[5] R. Engelking and R. Pol, Compactifications of countable-dimensional and strongly countable-dimensional spaces, Proc. Amer. Math. Soc. 104 (1988), 985-987.

[6] K. Nagami and J. H. Roberts, A note on countable-dimensional metric spaces, Proc. Japan Acad. 41 (1965), 155-158.

[7] E. Pol, Remarks on compactifications of metrizable spaces, Topology Appl. 15 (1983), 293-299.

[8] - The Baire-category method in some compact extension problems, Pacific J. Math. 122 (1986), 197-210.

[9] - Residuality of the set of embeddings into Nagata's $n$-dimensional universal spaces, Fund. Math. 129 (1988), 59-67.

Department of Mathematics

Ashikaga Institute of Technology

Faculty of Education

Ohmae 268-1

Saitama University

Urawa, Saitama 338-8570, Japan

Ashikaga, Tochigi 326-8558, Japan

E-mail: kimura@post.saitama-u.ac.jp

E-mail: mrst@ashitech.ac.jp

Received 26 June 2000;

in revised form 21 May 2001 